\title{
Small-angle X-ray scattering experiments and computer simulations to characterise anisotropy of activated carbons prepared from wood
}

\author{
András Wacha ${ }^{\mathrm{a}}$, Zoltán Varga ${ }^{\mathrm{a}}$, Ulla Vainio ${ }^{\mathrm{b}}$, Armin Hoell ${ }^{\mathrm{c}}$, Attila Bóta ${ }^{\mathrm{a}, *}$ \\ ${ }^{a}$ Hungarian Academy of Sciences, Chemical Research Centre. 59-67. Pusztaszeri út, Budapest, Hungary, H-1025 \\ ${ }^{b}$ Deutsches Elektronen-Synchrotron, Hamburger Synchrotronsrahlungslabor. Notkestraße 85, D-22607 Hamburg, Germany \\ ${ }^{c}$ Helmholtz-Zentrum Berlin für Materialen und Energie GmbH, Albert-Einstein-Str. 15, D-12489 Berlin, Germany
}

\begin{abstract}
Small-angle scattering was measured from activated carbon monoliths prepared from three different wood species (European beech, Pedunculate oak and Norwegian spruce). Substantial differences were found in the scattering images of axial and tangential slices, attributed to their characteristic phytogenic structures. The decrease in anisotropy in the nanostructured carbon matrix due to the activation process is also different among the three investigated types of wood. For quantitative characterization of the anisotropy and its decrease, the degree of alignment was calculated from the azimuthal scattering curves. A simple numeric model was constructed on the basis of direct information obtained by atomic force microscopy. Scattering patterns calculated from the model were compared with the experimental ones to explain the possible structural changes in the carbon skeleton.
\end{abstract}

\section{Introduction}

The functionality and area of application of activated carbons $(\mathrm{AC})$ is based on the properties of their complex pore structure, which is determined by the preparation process and the choice of precursors. It is of great importance to have the means to study - and possibly gain control over - the various parameters of the system. The sizes of the pores in activated carbons and sizes of cellulose nanocrystallites in plain wood are in the nanometer range, which can conveniently be studied using small-angle X-ray scattering (SAXS). Scattering experiments give statistically averaged information which can be further compared with theoretical results from simulations.

Because of its ubiquity and hierarchical microand nanostructure, one of the most commonly used precursors for activated carbon is wood. Activated carbons from wood are generally produced by the so-called "physical" activation process, consisting of two steps: carbonization (pyrolysis) and activation (by steam). During carbonization, the skeleton of carbonaceous material is formed with many

\footnotetext{
* Corresponding author

Email address: abota@chemres.hu (Attila Bóta)
}

structural and textural features resembling the original fibrous structure of the wood material. Several studies report details on the pyrolysis process [1]. Although only few of these are concerned with the structural relations of raw and carbonized wood, they agree that distinct features of the product can be attributed to the fibrous structure of the precursor $[2-5]$.

As a biomaterial, wood has to fulfil dual roles of static stabilization and transport of nutrients. Meeting both of these requirements is made possible by the layered structure and arrangement of wood fibres. The structure of wood on the superand sub-cellular length-scales has been extensively studied [e.g. 6, 7]. The wood tissue is composed mostly of elongated cells oriented along the stem axis, enclosing lumina. The origin of the mechanical stability lies in the ultrastructure of the cell wall [8-10]. The wood cell wall consists of cellulose fibrils that are embedded in a hemicellulose-lignin matrix, and wound up along the cell axis in a helical way. With respect to the spiral angle of these helices (also known as the microfibril angle, MFA), the cell wall can be classified into several layers [11]. The thickest one is the so-called " $\mathrm{S}_{2}$ ", its average MFA being $5-20^{\circ}$, depending on the species, 
origin, age and history of the tree. This angle is the primary parameter determining the mechanical strength of wood $[10,12]$.

The typical width of the cellulose microfibrils is reported to be $2.5-3.5 \mathrm{~nm}$, and their length lies in the range of a few millimetres [13-15]. On the packing of the microfibrils, different results are available. Some studies report random orientation of the cellwall components in the transverse plane of the $\mathrm{S}_{2}$ layer $[$ e.g. 16]. Others found the elementary cellulose fibrils to aggregate into larger fibers [17, 18]. The typical thickness of these so-called macrofibrils depends strongly on the origin and history of the wood specimen, but usually falls in the range of $10-30 \mathrm{~nm}[19,20]$.

The carbon matrix, which is created during carbonization of the fibrous wood, burns off partly in the activation step, giving place for the complex pore structure. The progress of this process determines the development of porosity [21]. First, small crystallites, or single layers of graphene gasify, resulting dominantly in the formation of micropores. Further activation makes whole crystalline groups burn off, while meso- and macropores evolve. Consequently, similarities between structure and texture of pyrolized precursors and ACs originating from anisotropic fibrils can only be observed at the beginning of the activation process.

Even though ACs are produced from different materials, their structures are similar: they are two-phase systems consisting of a carbon skeleton, which is usually composed of turbostratically packed graphene sheets [22], and voids, or pores, which are created mainly during the process of activation. This pore structure is the main cause of the good adsorption properties of activated carbons.

Small-angle X-ray scattering experiments are generally carried out using powdered activated carbon samples, in analogy to powder diffraction. This procedure does not give any information on the anisotropy of the sample. We have already investigated anisotropy of activated carbons originating from different types of wood using small-angle scattering and found that the anisotropy of twodimensional scattering patterns was characteristic for the fibrous phytogenic structure of the wood precursors [23, 24]. Considerable anisotropy was observed in monolith (cube) carbon samples from Pinus sylvestris, and the effect of this structure on the adsorption behaviour was examined by using SAXS [25].

In this paper we present measurements and inter- pretation of anisotropic small-angle scattering images obtained from activated carbons of different origin (European beech, Norwegian spruce and Pedunculate oak). The changes induced by pyrolysis and activation in the anisotropic structure of wood fibres are discussed in detail by means of a simple model consisting of polydisperse spherical units.

\section{Materials, methods and preliminary char- acterization}

\subsection{Preparation of the samples}

Activated carbon samples were prepared from three types of wood: European beech (Fagus sylvatica), Pedunculate oak (Quercus robur) and Norwegian spruce (Picea abies). Air-dried slats from trunks of mature trees (Sawmill of Eger Forestry Co, Hungary), where the thickness of early and late wood was less than a millimetre, were cut into small, regular cubes with an edge-length of approx. $10 \mathrm{~mm}$.

These wooden cubes were carbonized at $700{ }^{\circ} \mathrm{C}$ (in amounts of $200 \mathrm{~g}$ ) in a steel reactor flushed with nitrogen gas (flowrate: $50 \mathrm{dm}^{3} / \mathrm{h}$ ), for 2 hours. During this time, the length of the cube edges shrank by approx. 4-6 mm. Only a slight change in the shape of the monoliths was observed, in contrast to the literature [2]. This may be the consequence of the fact, that the wood we have used was matured for years before use and the wooden cubes were dried at $110{ }^{\circ} \mathrm{C}$ before the pyrolysis. No significant dimensional changes were observed however due to activation [26]. These carbonized specimens were activated (in amounts of $10 \mathrm{~g}$ ) in a rotary quartz reactor under steam flow of $18 \mathrm{~g} / \mathrm{h}$ at $900{ }^{\circ} \mathrm{C}$, diluted by nitrogen in a molar ratio of $1: 1$ [27]. The time of activation varied between 15 and 90 minutes, resulting in a wide range of burn-off (conversion of carbonized material), between 10$55 \%$. The loss due to mechanical abrasion could be neglected. The change in burn-off among different batches (same wood species and activation time) fluctuated within $1 \%$, while the specific surface (controlled by iodine-number) within $3 \%$, which proves the exceptional stability and controllability of the reactor [23].

The burn-off is shown in Fig. 1, as a function of the time of activation. It increases in a linear way for all types of carbonized wood as activation advances, indicating a pseudo-zero order conversion rate in a wide range of conversion from 0 up to $55 \%$. 


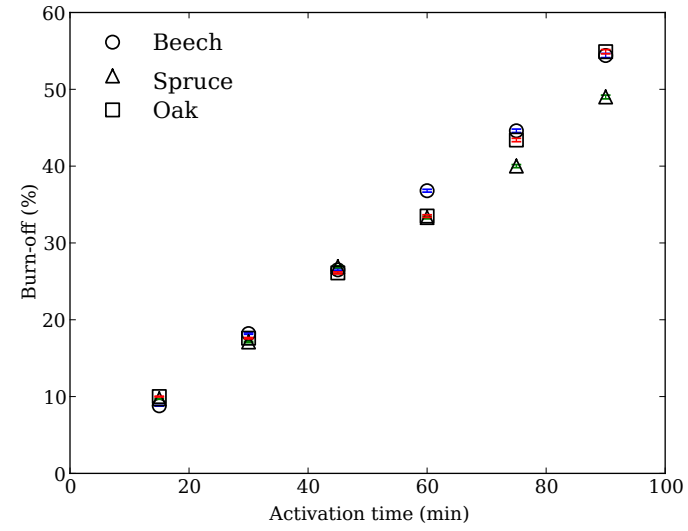

Figure 1: Burn-off of different carbon species due to activation. Error bars are smaller than the size of the markers.

Presumably, the decrease in mass is compensated by the increase in the area of the gas-solid interface due to the activation process. A further feature of the matrix is that also the porosity increases in a linear manner, while the outer shape and geometry of the cubes is not affected significantly.

It is worth noticing that the temperature and the time of carbonization strongly influences the structure of the matrix and the pore system as it was concluded by simultaneous adsorption and SAXS measurements in case of the shell of apricot stone (Prunus armeniaca) [28]. The high temperature of carbonization/pyrolysis $\left(850\right.$ or $1200^{\circ} \mathrm{C}$ ) results in a more compact carbon skeleton and the formation of an uniform pore system, as it was observed in the case of olive stone [21].

\subsection{Adsorption measurements}

For characterization of the adsorption capacity (e.g. specific surface area) iodine and nitrogen adsorption measurements were performed. Iodine adsorption (iodine number) tells about the internal surface of activated carbon. $1 \mathrm{mg}$ of adsorbed iodine is considered to represent $1 \mathrm{~m}^{2}$ of internal surface (according to the standard method DIN 53241). Nitrogen adsorption/desorption isotherms were used to determine the specific surface area. These isotherms were measured at the boiling point of liquid nitrogen by an AUTOSORB-1 (Quantachrome, Syosset, NY, USA) computer controlled automatic surface analyser and data processing system. Specific surface area was calculated according to the multi-point B.E.T. method.
The increase in the specific surface area during activation (Fig. 2) starts linearly, but levels off at higher values of the burn-off. The pyrolysed precursors have about $200 \mathrm{~m}^{2} / \mathrm{g}$ specific surface, offered by open pores. The higher the burn-off values are, the more the results of the two different absorption experiments differ.

The deviation from the linear behaviour clearly shows that at the beginning of activation mostly small pores are formed, resulting in a significant increase in the specific surface. In the region of higher conversions, larger pores are formed, as a consequence of the gasification of larger structural units, which can contribute to the increase of the specific surface only to a limited extent.

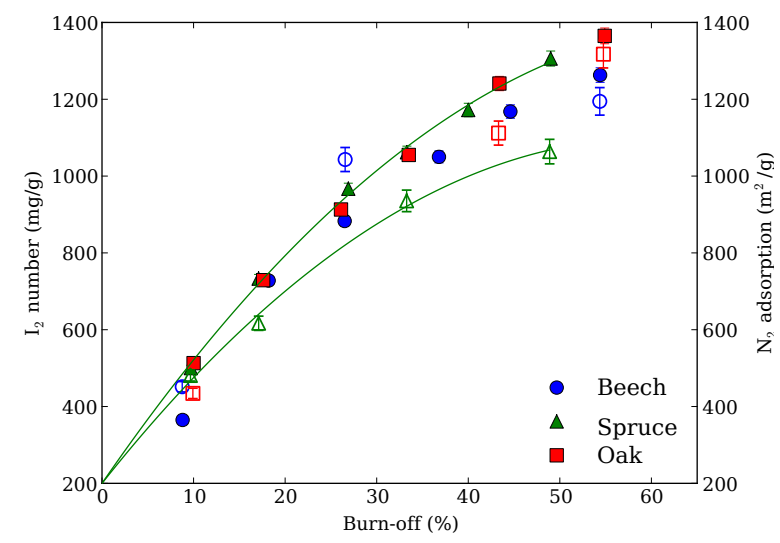

Figure 2: $\mathrm{N}_{2}$ (open symbols) and $\mathrm{I}_{2}$ (closed symbols) adsorption capacities of different AC specimens as a function of burn-off. Among the samples, spruce shows the largest difference between the results of the two methods, indicated by the fitted lines.

\subsection{Microscopy}

Atomic force microscopy (AFM) and scanning tunneling microscopy (STM) are good means to study the nanostructure of activated carbons [29]. AFM investigations of our samples were carried out using a Digital Instruments NanoScope III Atomic Force Microscope. To prevent damaging of the sample, tapping mode was used.

Atomic force micrographs of the plain carbonized samples (shown in Fig. 3) show that the texture of pyrolised wood is composed of several compact structural units. The size, shape and distribution (packing) of these varies from specimen to specimen. Beech has more or less spherical, largely poly- 

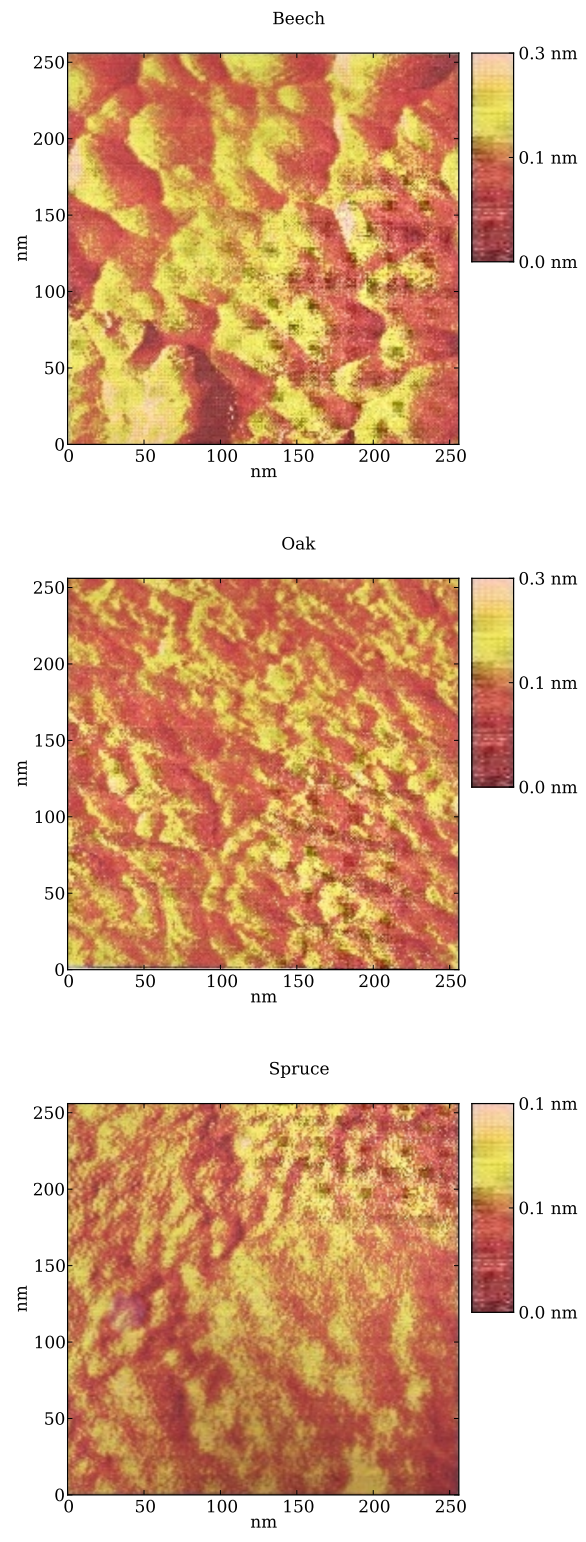

Figure 3: (Colour online) AFM images of carbonized wood samples.

disperse components in the size range of 40-60 nm, distributed randomly, while in spruce, these components exhibit some anisotropy and alignment in their shapes as well as in their distribution, extending in length from 20 to $100 \mathrm{~nm}$.

The scanning electron-micrographs (SEM, recorded with a Hitachi SEM-570 apparatus) of lateral cross-sections of carbonized samples are shown in Fig. 4. In these images the texture

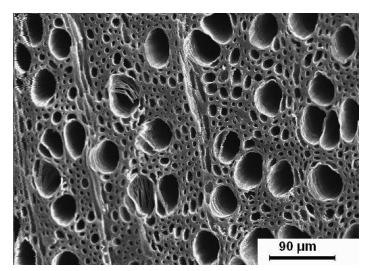

(a) Beech

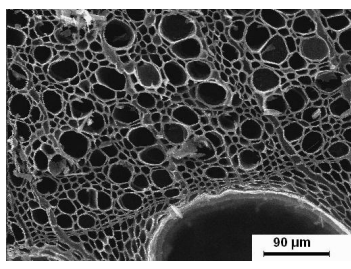

(b) Oak

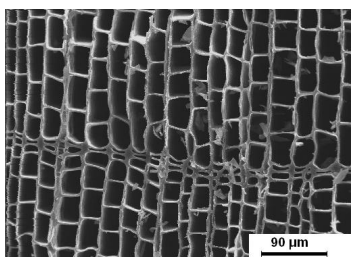

(c) Spruce

Figure 4: Scanning electron-micrographs of cross-sections of carbonized wood samples. White network corresponds to the carbonized remnants of the cell walls, while black voids are the lumina of wood cells.

of native wood can be recognised: the network of the cell walls is clearly visible. Beech and oak are composed of round cells of different size, dispersed randomly in the transversal plane. In contrast, spruce has nearly uniform, rectangular cells [e.g. 15]. Consequently, after carbonization, the remnants of the cell walls are expected to exhibit similar properties.

\subsection{Small-angle X-ray scattering}

Small-angle X-ray scattering measurements were performed at beamline 7T-MPW-SAXS of BESSYII, Berlin, Germany, and at beamline B1 of DORIS III, HASYLAB/DESY, Hamburg, Germany. On the first instrument, a monochromatized beam of photon energy $8311 \mathrm{eV}$ was used, the aperture before the sample being $0.5 \mathrm{~mm}$ horizontally and $0.4 \mathrm{~mm}$ vertically. Scattering patterns were recorded with a multiwire proportional chamber detector. The image was divided in $1024 \times 1024$ pixels, the pixel size being $0.207 \mathrm{~mm}$ in both directions. Two sample-to-detector distances were used, 3745 and $1376 \mathrm{~mm}$. In Hamburg scattering images were recorded with a Pilatus 1M (DECTRIS AG, Baden, Switzerland) detector, placed at a $885 \mathrm{~mm}$ distance from the sample. The monochromatized beam had size $1 \times 0.7 \mathrm{~mm}^{2}$ and photon energy of $18022 \mathrm{eV}$. 


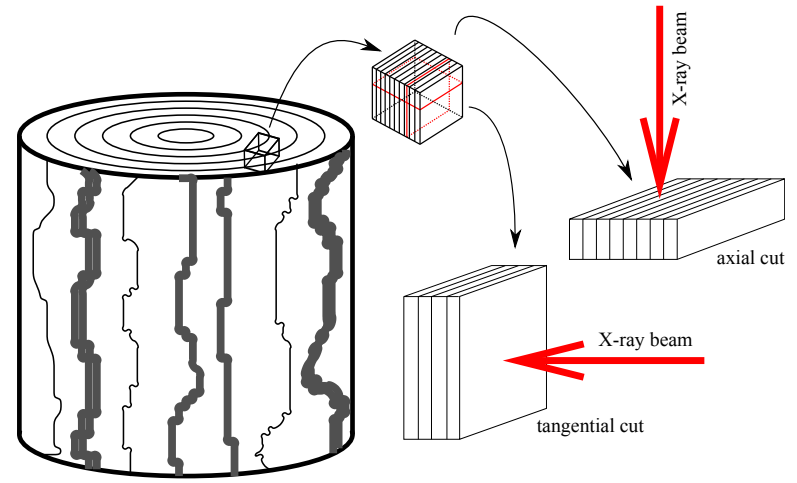

Figure 5: Tangential and axial cut of wood samples. Stripes mark the direction of growth-rings. Red arrows indicate the alignment of specimens with respect to the X-ray beam.

Approximately $1 \mathrm{~mm}$ thick radial and axial slices were cut from the carbon cubes by placing the cubes in the intersection of two orthogonal planes and using a very sharp razor blade. The slicing directions are shown in Fig. 5. The difference between early and late wood was clearly visible, even after activation, therefore the slicing of each cube and the alignment of the specimens in the X-ray beam was possible in both orientations, with respect to the tracheae. The two slicing directions are denoted by "tangential" and "axial", hinting on the orientation of the normal of the slicing plane with respect to the stem axis. The third ("radial") slicing direction was not considered, as small-angle X-ray scattering from radial and tangential sections of wood do not differ significantly [13].

For each activation time of each wood species, one tangential and one axial sample was used for SAXS measurements.

Two-dimensional scattering patterns were corrected for background, camera distortion and absorption effects by using the program packages specific to the measurement stations.

For quantitative analysis, sector averaged radial and stripe averaged azimuthal scattering curves were calculated. Azimuthal scattering curves were determined by evaluating numerically the integral:

$$
\tilde{I}(\chi)=\int_{q_{1}}^{q_{2}} I\left(q \cdot\left(\mathbf{e}_{\mathbf{x}} \cos \chi+\mathbf{e}_{\mathbf{y}} \sin \chi\right)\right) \mathrm{d} q
$$

where $\mathbf{e}_{\mathbf{x}}$ and $\mathbf{e}_{\mathbf{y}}$ are two orthogonal unit vectors in the image plane ( $x$ pointing down and $y$ right from the beam center) and $\chi$ is the azimuth angle [see e.g. 5]. q denotes the momentum transfer vector, its length being $q=4 \pi \sin \theta / \lambda$, where $\theta$ is the half scattering angle. Thus the integration area is a ring on the scattering pattern with $q_{1}$ and $q_{2}$ as inner and outer radii. To eliminate effects of illdetermined beam position, the origin of the image was adjusted by minimizing $\left|I(\chi)-I\left(\chi+180^{\circ}\right)\right|$ in the least-squares sense, making use thereby of the centrosymmetry of the scattering patterns, which follows from the centrosymmetry of the scatterers.

From these azimuthal curves, the azimuth angles of the two sets of maxima (detailed discussion in section 3.2) were determined. Sector averages were calculated along those preferred directions (angular size of the sectors was $5^{\circ}$ ), by masking out irrelevant detector pixels. The statistical quality of the averaged curves has been improved by taking advantage once again of the centrosymmetry of the scattering image. Finally, sector averages determined from different expositions of the same sample were summed to get the radial intensity on the largest possible $q$-range.

\subsection{Computer simulation}

As an aid to interpreting the SAXS images, computer simulations were carried out to determine the small-angle scattering properties of simple models emphasizing the anisotropic features of nanostructured carbonized wood. For the construction of these models different a priori considerations and direct experimental data were taken into account. First of all, the AFM images (Fig. 3) show that the matrix is composed of large structural units extending up to approx. $100 \mathrm{~nm}$. The changes occurring during activation influence the formation of micro and mesopores significantly, therefore these changes take place in the size range of 1-50 $\mathrm{nm}$. We have observed that the anisotropic scattering patterns vanish at about $q=0.1 \AA^{-1}$. After this value the Guinier scattering of small compact units can be seen (see later). The characteristic value of the radius of gyration falls into the size range of about $1 \mathrm{~nm}$, concluding that this is the lower size limit of our model.

The basic components were hard spheres, with smooth surfaces and a homogeneous electron density inside them. The radii of the spheres were sampled from a Gaussian distribution. The choice fell on spheres for three reasons. Firstly, the general model of the nanostructure of ACs assumes the matrix to be built up from small, isotropic "crystallites" of turbostratically packed graphene sheets. Secondly, the scattering form factor of a sphere can be computed analytically. Thirdly, building 
anisotropic structures from isotropic components enables one to simulate the loss of anisotropy by pore formation.

The model specimens were constructed according to the following procedure. Firstly, rod-like structures responsible for anisotropy (fibrils) were built up by placing the spherical units after each other in such a way that neighbouring spheres touch each other. The incoming angle of each new sphere was sampled uniformly in a cone with its axis being the straight line connecting the centres of the two most recently added spheres.

Two extreme types of fibrils were investigated: "straight" and "uneven". Each of them was constructed from 15 spheres. For straight fibrils, the average radius of the spheres was $1.7 \mathrm{~nm}$ with a standard deviation $0.25 \mathrm{~nm}$, and the half-aperture of the construction cone (follow angle) was $5^{\circ}$. Uneven fibrils were meant to be rougher, which was accomplished by setting the standard deviation of the radii to $0.75 \mathrm{~nm}$ (while keeping the average at $1.2 \mathrm{~nm}$ ) and the follow angle to $45^{\circ}$. The average length of the individual fibres was approximately $160 \mathrm{~nm}$ for straight fibrils and $43 \mathrm{~nm}$ for uneven ones. The lateral extent of these fibrils falls in the range of $2.5-3.5 \mathrm{~nm}$, in accordance with the literature on the thickness of cellulose microfibrils in wood [13-15].

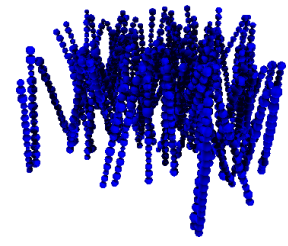

(a) Straight fibrils

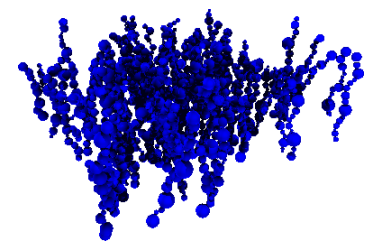

(b) Uneven fibrils

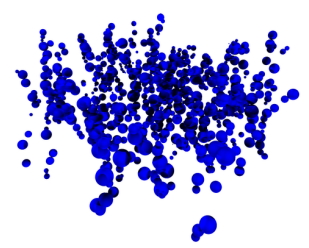

(c) Activated form

Figure 6: Models for activated carbons. The length of the fibrils is approx. $30 \mathrm{~nm}$.

In the second step, each rod was tilted by a random angle (sampled from a Gaussian distribution) from the vertical direction, then rotated around the vertical axis at random (the angle of rotation followed a uniform distribution on $\left[0^{\circ}, 360^{\circ}[)\right.$. Lastly, all such transformed rods were packed together by moving them one by one in the horizontal plane towards the origin until collision, to yield model specimens, a few representatives of which are shown in Fig. 6. The mean of the tilt angle is denoted later on as "nanofibril angle" (NFA), as a distinction from the microfibril angle (MFA) in native wood.

The effect of the activation process was simulated by removing a given amount of spherical units from each sample at random, assuming thereby the activating agent to be able to penetrate the sample homogeneously. This method was expected to simulate the opening of mesopores, observed during activation of pyrolized wood.

Small-angle scattering patterns of the model scatterers described above were calculated according to a generalized version of the Debye formula, i.e. scattering of a particulate system, without averaging over the macroscopic orientation of the sample [30]:

$$
I(\mathbf{q})=\sum_{j} \sum_{k} F_{j}(q) F_{k}(q) \cos \left(\mathbf{q} \cdot \mathbf{r}_{j k}\right)
$$

where $F_{j}(q)$ is the form factor of the $j$-th sphere, $q$ is the length of the momentum transfer vector $\mathbf{q}, \mathbf{r}_{j k}$ is the distance vector of the centres of sphere $j$ and $k$, and sums are done over all spheres. For creating scattering models and calculating their scattering, a purpose-made program suite was used. The computing core was implemented in $\mathrm{C}$ and Cython, while the controlling procedures were Python scripts ${ }^{1}$.

Scattering patterns were calculated in a transmission set-up, with a $256 \times 256$ matrix for a flat position-sensitive detector $\left(10 \times 10 \mathrm{~cm}^{2}\right.$ in area). The theoretical X-ray source was an infinitely small point-collimated beam of completely monochromatic $\mathrm{Cu}-\mathrm{K} \alpha$ radiation $(\lambda=1.54 \AA)$, hitting exactly the centre of the detector. Smearing effects caused by finite beam or pixel sizes were not accounted for. Adsorption effects were also neglected. The distance from the detector plane to the sample was $5.75 \mathrm{~m}$. The axis of the theoretical X-ray beam was lying in the horizontal plane of the model specimens, thus the calculated patterns correspond to those measured from the tangential cuts of activated carbon samples.

\footnotetext{
${ }^{1}$ For more information, please contact the authors at wacha@chemres.hu
} 
The way of computing the small-angle scattering patterns (i.e. the generalized Debye-formula) posed limitations on the size of the model specimens, as the CPU time for the calculation scales with $O\left(N^{2}\right)$. A good compromise between model size and calculation time was when each model specimen consisted of 100 fibrils, constructed from 15 spheres each, thereby giving 1500 spheres in total per model specimen. To rule out artefacts caused by finite size and uncontrolled parameters, around 20-35 instances were created at random for each simulation (identified by fibre type and NFA), and their scattering patterns were averaged. This way the images became smoother, but no matter how large number of random instances is chosen, this type of modelling (direct calculation of scattering patterns from sphere structure) is not capable of yielding the quality of real-world SAXS experiments, where averaging is done over ensembles larger by several orders of magnitudes.

Another consequence of the finite size is the lack of fractality. To achieve fractal-like power-law scattering curves, similar structure should exist at several orders of magnitude, which cannot be achieved with the present model.

Another simplification is the complete absence of ordering in the lateral plane (with respect to the trunk axis), which rendered the model unusable for studying features seen in axial samples. However, lateral ordering is expected to have only little impact on scattering properties of tangential cuts.

\section{Small-angle $\mathrm{X}$-ray scattering: results and discussion}

\subsection{Two-dimensional small-angle scattering pat- terns}

\subsubsection{Axial cut}

Scattering images obtained on samples cut in the axial direction are displayed in Fig. 7. In this cutting direction, scattering from carbonized samples differs strongly: beech exhibits completely isotropic scattering, oak shows elongated features, while the patterns measured from spruce contain a cross-like shape.

The morphological features seen in SEM micrographs presented before (in Fig. 4) give an explanation to these differences. Because of the rectangular cell walls of spruce, the projection of its microstructure onto the axial cutting plane exhibits two nearly

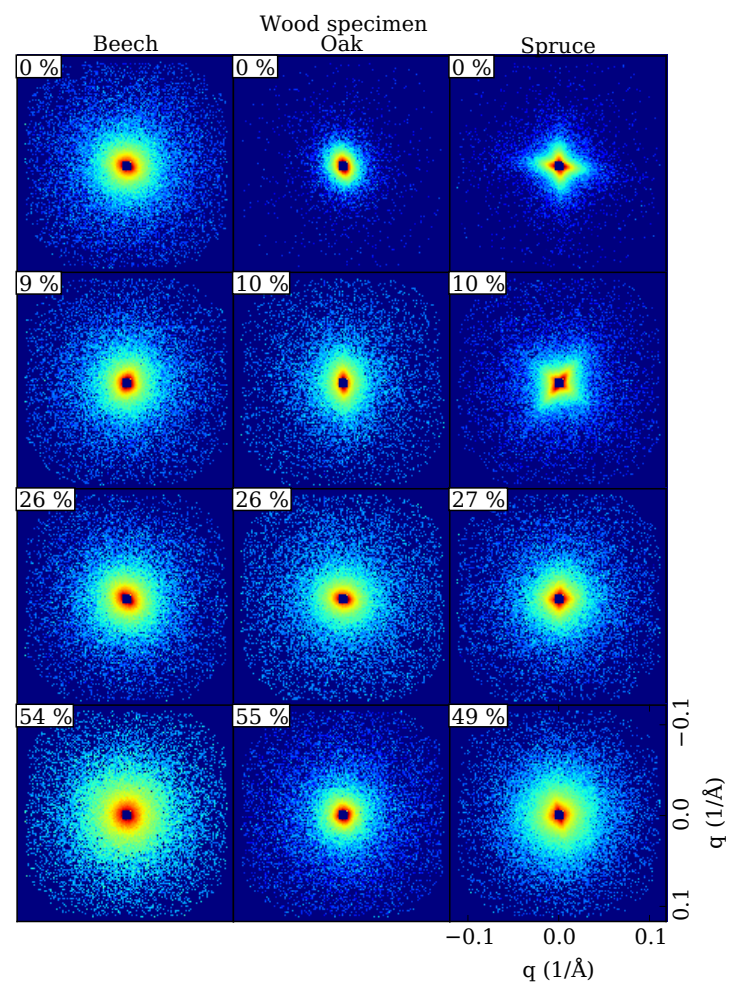

Figure 7: (Colour online) Measured SAXS images of the wood-derived AC samples, axial cut. The stem axis is orthogonal to the image plane. Labels in the upper left corners of the images indicate the burn-off ratio. The colour scale is logarithmic.

orthogonal preferred directions in the corresponding microscopic image. The "shafts" of the crossshape in the SAXS pattern correspond to these directions, i.e. the horizontally elongated structures in the projection of the carbonized fibrils onto the cut plane (parallel to the detector plane) cause vertical widening of the scattering pattern, while horizontal widening is the effect of vertical structures. In a similar way, the slightly elongated or isotropic shape of the SAXS patterns of oak or beech, respectively, can be attributed to the irregular and polydisperse shape and unordered packing of their cells in the axial plane.

At the beginning of activation (15 minutes, second row in Fig. 7) the characteristic patterns persist - except for a rotation by an arbitrary angle around the axis of the X-ray beam. The cause of this rotation is the undefined alignment of the sample in the plane orthogonal to the X-ray beam.

As the activation process extends further, 
anisotropy diminishes, although in the case of spruce- and oakwood, anisotropy remains to a small extent even after 90 minutes of activation (last row in Fig. 7). Apart from this, small streaks can be distinguished near the beam-stop area $\left(q<0.01 \AA^{-1}\right)$. This feature originates from the structure of the sample, and can be attributed to anisotropic micro-cracks in the carbon texture. This type of anisotropy survives even 90 minutes of activation (approx. 50\% burn-off). Comparing the changes due to activation at larger and smaller values of $q$, it can be concluded that anisotropy exists at more than one independent length scales.

\subsubsection{Tangential cut}

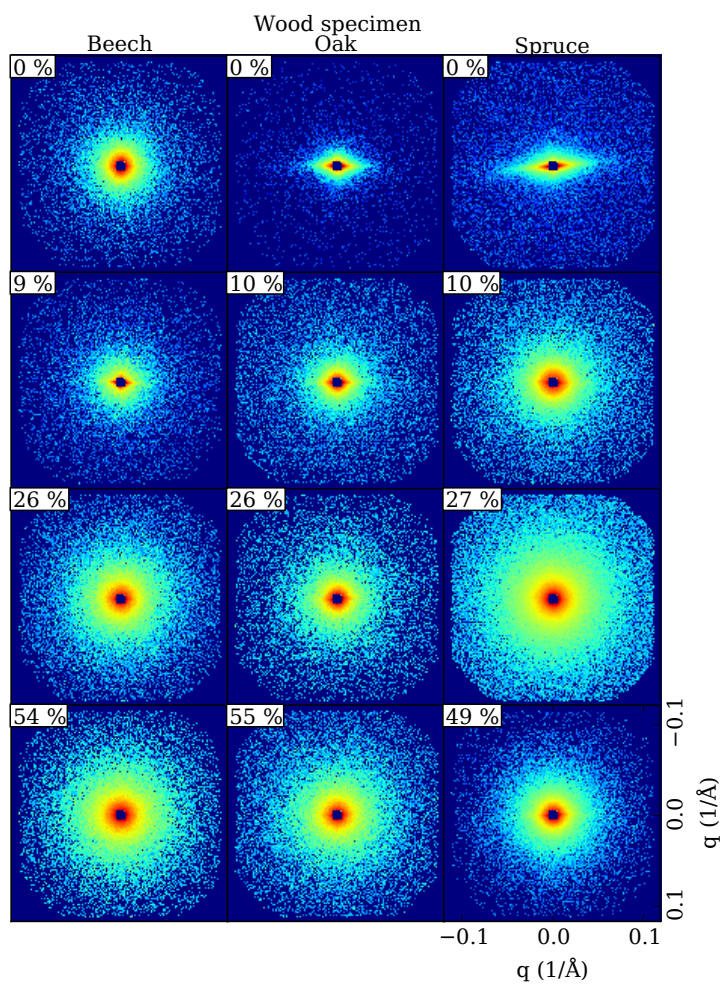

Figure 8: (Colour online) Measured SAXS images of the wood-derived AC samples, tangential cut, sample-todetector distance was $3745 \mathrm{~mm}$. The stem axis is vertical. Labels in the upper left corners of the images indicate the burn-off ratio. The colour scale is logarithmic

SAXS images from tangential cuts are shown in Fig. 8. All specimens were aligned in such a way that the stem axis was nearly parallel to the vertical direction on the scattering image, because preferred directions on the surface of the specimens were clearly visible to the naked eye, in contrast to axial cuts.

The different anisotropy in scattering patterns from carbonized wood samples, depending on the species of wood, is clearly visible. Spruce exhibits the largest anisotropy, while beech the least. During activation, these anisotropic features decrease for every wood specimen, in a similar way as found in axially cut samples and the scattering images become circularly symmetric, apart from the contributions of micro-cracks, which are still present even after 90 minutes of activation, as in the case of axial samples. As these streaks are horizontal, they may be identified as the contributions of vertical micro-cracks in the samples.

The scattering patterns of plain carbonized wood exhibit similar features as native wood does $[5,13$, 14]. In those, nearly horizontal streaks in the range $0.1<q<0.4 \AA^{-1}$ are usually caused by the approximately vertical alignment and elongated shape of (micro)fibrils [31]. For smaller angles (below $q=0.05 \AA^{-1}$ ) scattering was found to be dominated by voids [32]. However, these features of native wood can undergo drastic changes during carbonization and activation.

\subsubsection{Computer simulation}

Small-angle scattering images of the scattering models described in section 2.5 are displayed in Figs. 9 and 10. The nanofibril angle, i.e. the random tilt angle of the fibres with respect to the stem axis was taken from a Gaussian distribution of standard deviation $5^{\circ}$, centred at 0,15 or $30^{\circ}$. Microfibril angles are known to fall mostly into this range according the literature. Burn-off values were chosen to correspond to those of activated wood.

The first lines of the figures show the scattering patterns of the initial, i.e. not activated models. Each image exhibits two sets of streaks. Horizontal, continuous ones, which split up with increasing NFA are caused by the orientation of individual fibrils with respect to the vertical axis, while vertical, more discrete ones can be understood as effects of the chain-like packing of the spheres in the fibrils. Indeed, for uneven fibrils, this feature is less expressed, because of the ordering of the spheres in the fibrils is less complete.

Increasing the NFA, SAXS patterns show azimuthal broadening and splitting of the horizontal streak signal. This effect bears resemblance to the broadening caused by the variation in the cellulose microfibril angle on which various authors give both 


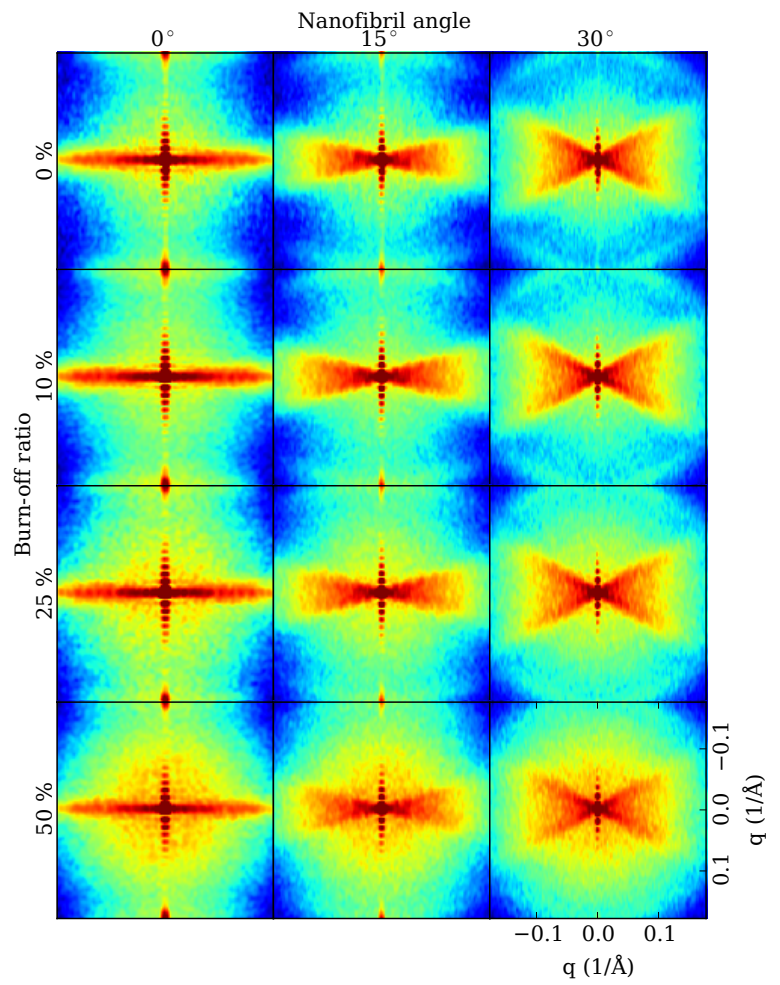

Figure 9: (Colour online) Calculated 2D X-ray scattering of straight models. The colour scale is logarithmic.

theoretical and experimental evidence $[12,33]$. For the uneven model, this broadening is also present, but not so well-marked, and the radial extent of the butterfly pattern is smaller (see Fig. 10).

Tendencies similar to those observed in the SAXS patterns of activated tangential samples can be observed here as well. As more and more spheres are burnt off, an isotropic scattering signal appears at larger angles, originating from distinct compact scattering units, simultaneously decreasing the influence of fibrous structures on the scattering image. Vertical streaks remain approximately intact. In contrast to real carbonized wood, horizontal streaks remain visible even for large amounts of pores in case of straight models. In this sense, structures composed of uneven fibres, especially with $0^{\circ}$ NFA, are more accurate models for the anisotropy observed in wood-based activated carbons.

\subsection{Quantitative characterization of anisotropy}

Azimuthal scattering profiles-i.e. intensity vs. $\chi$, the azimuth angle - are more suitable for characterization of anisotropic scattering properties of

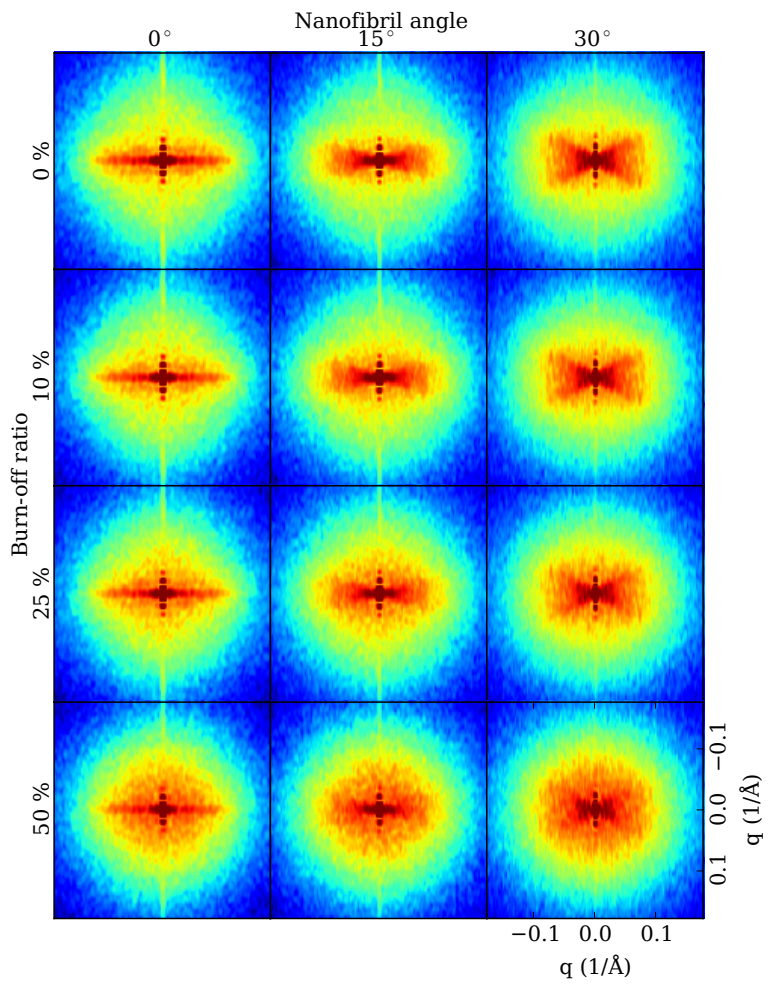

Figure 10: (Colour online) Calculated 2D X-ray scattering of uneven models. The colour scale is logarithmic.

both ACs and their theoretical models. Azimuthal curves were determined from the two-dimensional scattering patterns of tangential and axial AC samples at the $q$-range of the end of the horizontal streaks in plain carbonized spruce and oak $\left(0.024 \AA^{-1} \leq q \leq 0.048 \AA^{-1}\right)$. Carbonized beech also has substantial scattering intensity in this region. The curves themselves are shown in Fig. 11. The curves are periodic with period $180^{\circ}$, as a consequence of the centrosymmetric nature of the scatterers. The tangential sections of ACs produced from spruce and oak exhibit one set of peaks (near 90 and $270^{\circ}$ ), decreasing in height as the activation process proceeds. These correspond to the horizontal streaks in the respective scattering patterns. The remaining anisotropy at higher activation (especially in the case of spruce) is the manifestation of the scattering from micro-cracks, mentioned before.

Curves determined from the axial cuts of sprucederived ACs exhibit two sets of peaks, caused by the cross-like shape seen in the scattering patterns. The angular displacement of these peaks is not ex- 

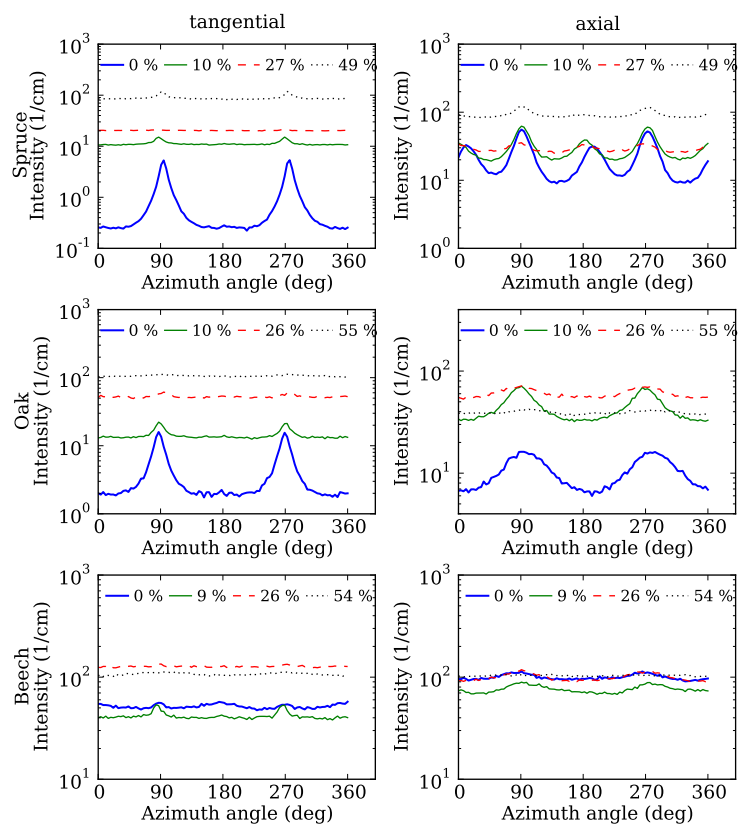

Figure 11: Azimuthal scattering curves for activated wood samples (in the range $0.024 \AA^{-1} \leq q \leq 0.048 \AA^{-1}$ ). The burn-off is indicated for each curve. Axial curves were shifted along the abscissa to eliminate the effects of the undefined macroscopic orientation of the specimens (no vertical scaling was done).

actly $90^{\circ}$, as the rectangular cell-shape would suggest [12]. The cause for this aberration may be that the cut plane was not completely orthogonal to the stem axis, making cut surfaces of the cells to be slanted parallelograms instead of rectangles.Similarly to tangential curves, the height of these peaks decreases during activation, leaving only one pair of peaks at the highest burn-off, which originate from micro-cracks in the texture of the samples.

Azimuthal curves obtained from axially cut ACs from oak have only one set of peaks, due to the different cross-sectional shape of the cells. They decrease in intensity during activation in a similar manner as in spruce-derived ACs.

Beech however exhibits different characteristics. As seen from the scattering patterns, it has but a very little anisotropy even at the beginning of activation. Beside the horizontal streaks $(\chi=90$ or $\left.270^{\circ}\right)$, another set of peaks can also be observed in the vertical direction $\left(\chi=0\right.$ or $\left.180^{\circ}\right)$ on the tangential curves, although only to a rather small extent. This set vanishes at lower burn-off than the horizontal streaks do during activation.

Azimuthal scattering patterns of the model specimens were calculated directly by a modified version of equation (2) and are shown in Fig. 12. To reduce effects of finite sample size, azimuthal curves calculated at 10 equidistant points of $q$ in the range of $[0.1,0.12] \AA^{-1}$ were averaged. Before plotting, all curves were smoothed by convolution with a $14^{\circ}$ wide Hanning window.
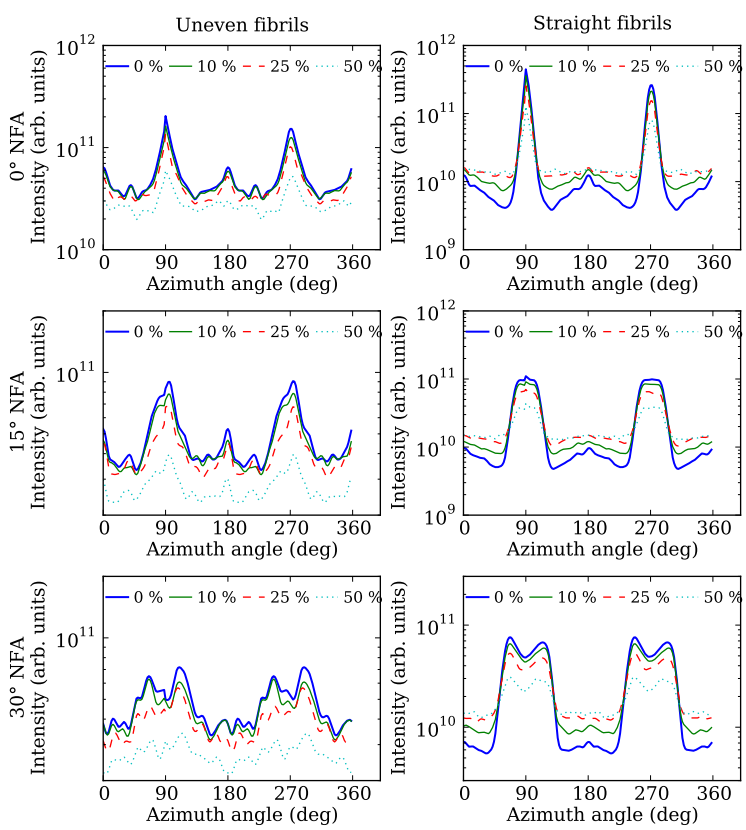

Figure 12: Azimuthal scattering profiles of the model specimens.

These curves have many common features with the measured ones. Large peaks (around 90 and $270^{\circ}$ ) originate from the broadening of the horizontal streaks, opening of the "butterfly wings" in the scattering patterns. Smaller ones at 0 and $180^{\circ}$ correspond to the vertical streak in Figs. 9 and 10, caused by the vertical packing of spherical subunits. It becomes clear that broadening of the previous ones can be attributed to the separation of two peaks in azimuth angle, which correspond to two extremal tilts (NFA) of the orthogonal projections of the fibres in the plane of the detector.

As seen in the measured patterns, activation, or pore formation reduces anisotropy. In the calculated curves, the same tendency can be observed. In contrast to measurements however, here the peaks at 0 and $180^{\circ}$ vanish faster, as a consequence of the 
difference of the effects causing them.

Based on these results, a qualitative comparision can be made between real ACs and the models. Generally, simulated specimens with uneven fibrils describe features of activated carbons better than their straight counterparts. Especially, the smallangle scattering patterns and azimuthal intensity profiles of the NFA $=0^{\circ}$ model series exhibit similar properties as of tangentially cut spruce and oak derivatives.

The most prominent feature found in both SAXS experiments and simulations is the disappearance of anisotropic features in scattering images due to activation. This can plausibly be attributed to the gasification of entire carbon crystallites in the reach of the activating agent, thereby causing the formation of mesopores, which in turn break the fibres into smaller components, reducing anisotropy.

For quantitative characterization, different measures can be devised. The most simple parameter is the degree of alignment $(\rho)$, introduced by Fratzl et al. [34]. It can directly be obtained from azimuthal scattering curves, even in relative intensity, as the ratio of the total peak area above the constant baseline and the integral of the curve with respect to the azimuth angle. In the case of isotropic scatterers, the net peak area over the baseline is zero, therefore the degree of alignment is zero in that case, and increases with increasing anisotropy. If the sample is "totally anisotropic", i.e. the baseline is at zero, one gets $\rho=1$, which is the highest possible value.

Fig. 13 shows the dependence of the degree of alignment on the burn-off for both tangential and axial cutting directions of wood-derived ACs. These results are in concordance with the previous findings. For both tangential and axial cuts, the degree of alignment in oak and spruce decrease with the burn-off, while in beech it remains small throughout the activation process. A profound difference can be observed between tangential and axial cuts. In the first case, a dramatic loss of alignment is found in the first 15 minutes of activation (cca. $10 \%$ burn-off), after which anisotropy changes only little. As at the beginning of the activation process mostly micropores are formed, it may be concluded that micropores contribute to the loss of anisotropy in the tangential direction significantly. In the axial cutting direction however $\rho$ decreases almost linearly for these two wood species. Maybe the anisotropy defined by the cell cross-sections in the precursor materials is more ro-
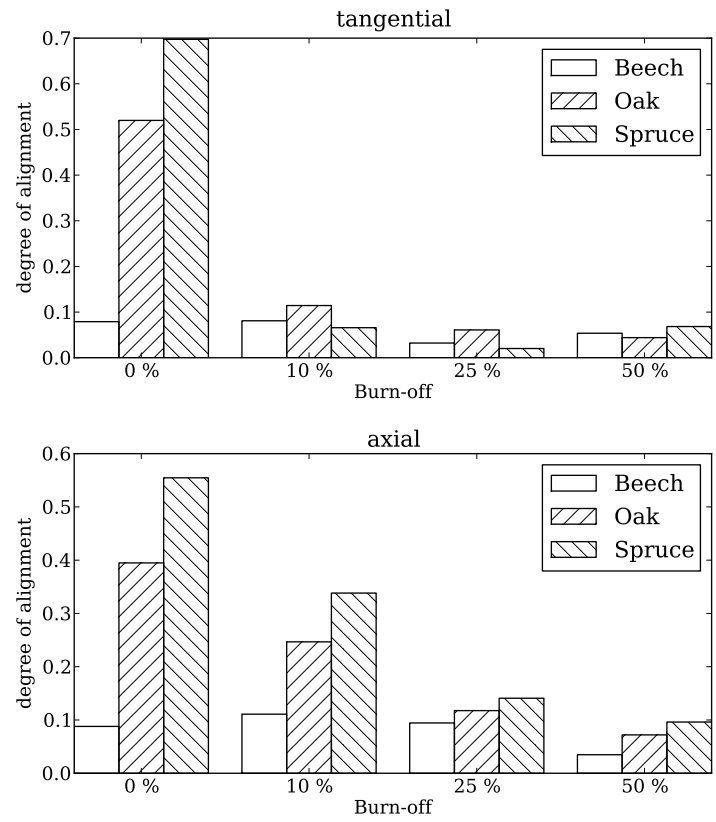

Figure 13: Anisotropy parameters for different specimens. Burn-off values were rounded to fives.

bust than the vertical, fibril-originated features.

Different tendencies can be observed in the corresponding graphs obtained from model specimens (Fig. 14). The decreasing nature of $\rho$ is retained, especially in straight fibrils, however the decrease is not so dramatic as found in spruce and oak. This can be attributed to the way of simulating the burnoff, namely assuming the activation agent to penetrate the whole sample uniformly, causing the formation of unimodal pores. The dependence of $\rho$ on the nanofibril angle is more complex and changes with the burn-off.

\subsection{Analysis of the scattering curves}

To elucidate the meaning of the features observed in the measured two-dimensional SAXS patterns and to estimate the extent of anisotropy, sector averages of the tangential patterns have been calculated according to the procedure described in section 2.4. For each image, three curves were calculated: one sector average along the horizontal streak (absolute maximum of the azimuthal curve), one along the vertical streak (second largest maximum in the azimuthal curve, nearly $90^{\circ}$ apart from the absolute maximum) and finally a full-circle average of the scattering patterns. These curves are 

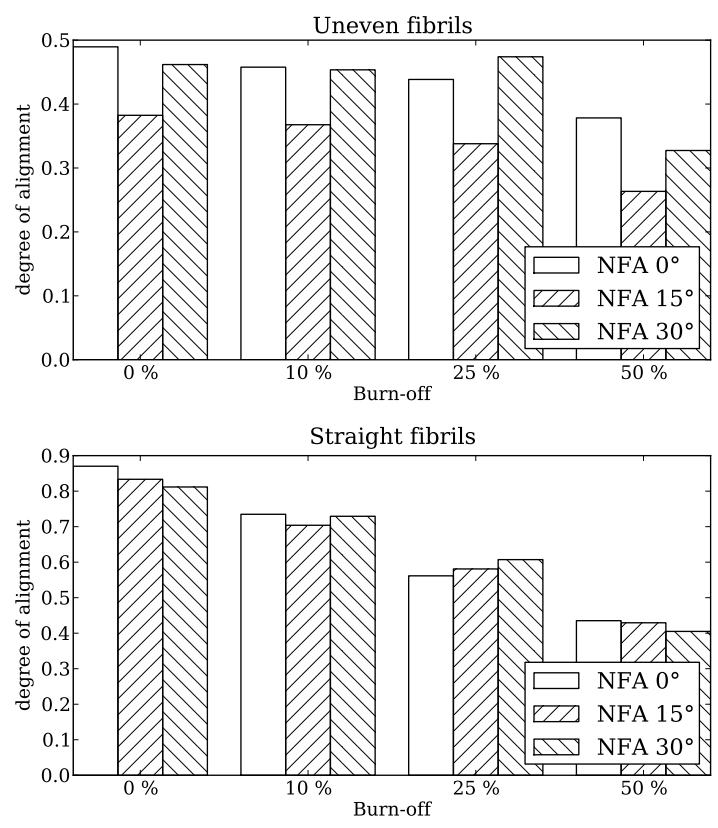

Figure 14: Anisotropy parameters of the models.

shown in Fig. 15.

Each curve starts with a power-law scattering region corresponding to the scattering of a fractal system, which is followed by one or more Guinier-like knees, and ends with a second power-law [35-37]. Comparing different curves of the same scattering specimen the extent of anisotropy can be determined in terms of $q$. From the Figure it is clear that anisotropic properties are limited to the first fractal-region (ending at $q \approx 0.1 \AA^{-1}$ ). It may be said therefore that this region can be attributed to the fractal properties of remnants from microfibrils. It is also noteworthy that vertical sectors approach the full-circle averages faster than horizontal ones during activation, i.e. the vertical anisotropy vanishes faster than the horizontal.

More accurate results can be obtained by fitting the horizontal and vertical sectors by appropriate model functions. Scattering curves with a knee (all curves with the exception of the highest activated samples and the second-highest activated sample of beech), were able to be fitted well by the sum of a power-law and an empirical Guinier-Porod curve [38]:

$$
I(q)=A q^{\alpha}+\Phi\left(q, G, R_{g}, \beta\right)
$$

The second term, the compound Guinier-Porod

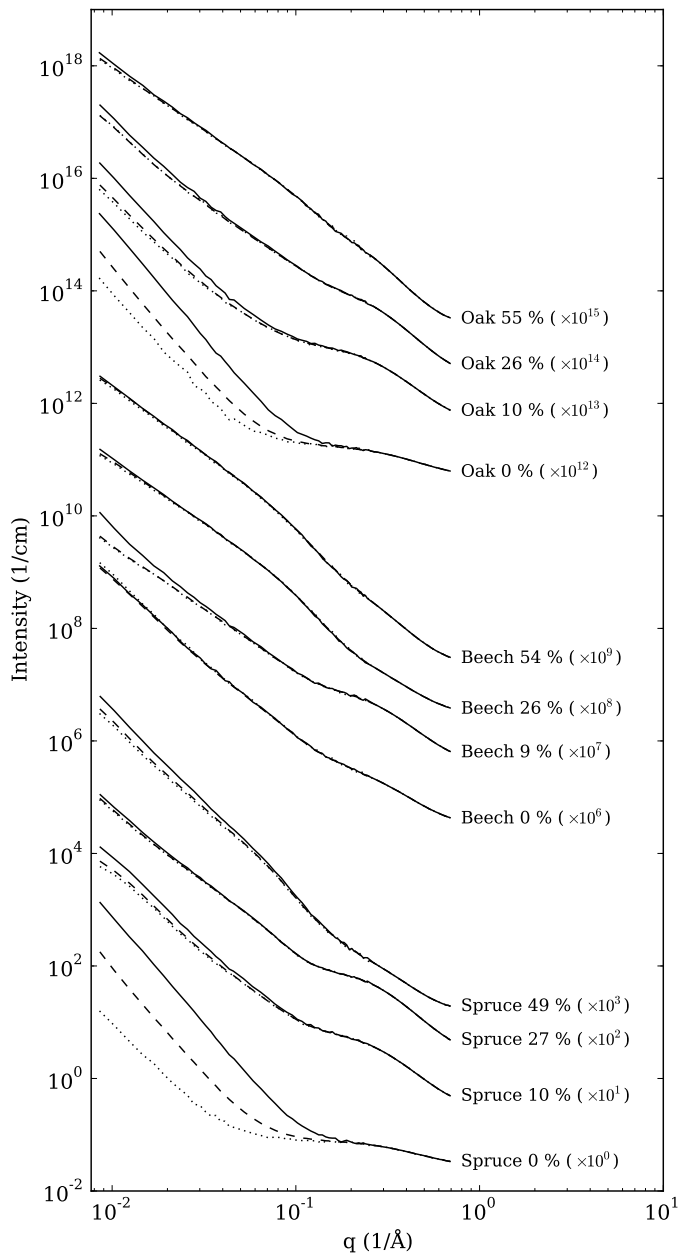

Figure 15: Slices of scattering images (tangential cuts) along the horizontal $(-)$ and vertical $(---)$ directions, as well as the full azimuthal averages $(\cdots)$. For the sake of visibility, curves were multiplied to avoid overlaps (multiplication factor for each curve is indicated).

curve is defined as:

$$
\Phi\left(q, G, R_{g}, \beta\right)=\left\{\begin{array}{lll}
G e^{-\frac{R_{g}^{2} q^{2}}{3}} & \text { if } & q \leq q^{*} \\
B q^{\beta} & \text { if } & q \geq q^{*}
\end{array}\right.
$$

where the parameters $q^{*}$ and $B$ are defined by $G$, $R_{g}$ and $\beta$ through constraints of smoothness in $q^{*}$. In other words, a Guinier scattering law is coupled to the power-law region after it, whereby one fitting 
parameter (the scaling factor for the power-law) can be spared. The other curves (with exception of the highest activated oak sample) could be fitted by a "reversed" version of this fitting function, i.e. where the Guinier function was attached to the end of the low- $q$ power-law with similar constraints of smoothness. The fitting was carried out on the range of $0.0086 \leq \AA^{-1} q \leq 0.576 \AA^{-1}$. Fitted curves are not displayed because of the perfectness of the fits (the coefficient of determination $\left(R^{2}\right)$ was better than 0.995 in all cases), and to retain the readability of the graph. The relevant structural parameters are displayed in Tab. 1.

From the Guinier ranges, radii of gyration have been determined. The growth of these radii can be observed in all curves, which is a consequence of the formation and growth of small, partially crystallized basic structural units [39]. Another interpretation is that the Guinier scattering of microor mesopores can be observed, due to the Babinet principle. The first explanation is supported by the fact that because of the low temperature of pyrolysis, the carbonization of the samples are not complete and continues during the activation process carried out at a higher temperature. The second one is plausible because of the formation and growth of the pores during steam activation.

In beech- and spruce-derived ACs a dramatic jump is observed in the Guinier radii, as another Guinier range appeared in the scattering curves, corresponding to mesopores. Parallelly, the impact of the other Guinier region decreases, which may be the consequence of the burn-off of whole carbon crystallites or the fusion of micropores. Oak exhibits similar features at the highest activation, where both Guinier knees can be observed, although very weakly, ill-suited to fit them with significance. The radii of mesopores increase with activation similarly those of micropores or partially crystallized structural units.

Power-law scattering in ACs is usually caused by surface, mass or pore fractal structure [40,41]. The general formula for the scattering of fractal systems is $I(q)=A q^{D_{s}-2\left(D_{m}+D_{p}\right)+6}$, which simplifies to $I(q)=A q^{D_{s}-6}$ for surface fractals $\left(D_{m}=D_{p}=3\right)$ and $I(q)=A q^{-D_{m}}$ or $I(q)=A q^{-D_{p}}$ for mass or pore fractals $\left(D_{p}=D_{s}=2\right.$ or $\left.D_{m}=D_{s}=2\right)$, respectively [42]. Surface fractals can be discerned from the other two "bulk" fractals by just analysing the exponent. On the other hand, telling mass and pore fractals apart needs knowledge from other sources.
ACs prepared from beech behave as mass or pore fractals at the low- $q$ range even without activation, corresponding to a porous structure, which describes its inherently weak anisotropy. The fractal dimension decreases during activation both in horizontal and vertical sectors, as a consequence of pore formation. Samples derived from oak start as surface fractals, turning later into bulk fractals, in a similar way as other authors found [41]. However, this conversion starts in our case with the increase of the surface fractal dimension, which may be a consequence of the fact that crystallization of the char proceeds during activation. After the conversion however, the same decrease of the bulk fractal dimension is observed as in beech.

Spruce exhibits the same tendency, except that at highest activation it turns once again into a surface fractal in both horizontal and vertical direction. Maybe pore formation goes to that extent that bulk fractality vanishes.

The ratio of the scaling factors of the low- $q$ power-law and the following Guinier range can act as a rough measure of the contribution of micro- or mesopores in scattering. These parameters can be found in the rightmost column of Tab. 1. For beech a growing tendency is observed, as a consequence of further crystallization or micropore formation. In spruce and oak on the contrary, this parameter decreases during activation. The cause of these differences may be the different relative evolution of the pore system and the carbon skeleton.

Simulated models on the other hand are not applicable for such treatment. Although they are good enough to describe anisotropic scattering features found in our AC samples, their construction method precludes the possibility of fractal structures because of the lack of the same structure on several length scales. A possible improvement would be to substitute the current spherical units with closely packed smaller ones. This would also allow for the more close modelling of microand mesopore formation by controlled removal of spheres.

\section{Conclusions}

Structural features - especially anisotropy - are retained in activated carbons prepared from the three typical European wood species investigated. Two-dimensional scattering images differ profoundly with respect to precursor species and slicing direction. Moreover they change differently as 


\begin{tabular}{|c|c|c|c|c|}
\hline Burn-off & $\begin{array}{c}\text { Low- } q \\
\text { exponent }\end{array}$ & $\begin{array}{c}\text { Low- } q \text { fractal } \\
\text { dimension (type) }\end{array}$ & $\begin{array}{l}\text { Radius of } \\
\text { gyration }[\AA]\end{array}$ & $\begin{array}{l}\text { Relative weight } \\
\text { of Guinier scattering }\end{array}$ \\
\hline \multicolumn{5}{|c|}{ Beech, horizontal sector } \\
\hline $0 \%$ & $-2.88 \pm 0.01$ & $2.88 \pm 0.01$ (mass/pore) & $4.57 \pm 0.02$ & $191 \pm 4$ \\
\hline $9 \%$ & $-2.71 \pm 0.01$ & $2.71 \pm 0.01$ (mass/pore) & $5.08 \pm 0.01$ & $306 \pm 8$ \\
\hline $26 \%$ & $-2.49 \pm 0.01$ & $2.49 \pm 0.01$ (mass/pore) & $24.13 \pm 0.11^{a}$ & $1870 \pm 80^{a}$ \\
\hline $54 \%$ & $-2.38 \pm 0.02$ & $2.38 \pm 0.02($ mass $/$ pore $)$ & $27.04 \pm 0.23^{a}$ & $1840 \pm 80^{a}$ \\
\hline \multicolumn{5}{|c|}{ Oak, horizontal sector } \\
\hline $0 \%$ & $-3.90 \pm 0.01$ & $2.10 \pm 0.01$ (surface) & $3.13 \pm 0.01$ & $8400 \pm 200$ \\
\hline $10 \%$ & $-3.28 \pm 0.01$ & $2.72 \pm 0.01$ (surface) & $5.37 \pm 0.01$ & $4100 \pm 100$ \\
\hline $26 \%$ & $-2.68 \pm 0.01$ & $2.68 \pm 0.01(\mathrm{mass} /$ pore $)$ & $5.70 \pm 0.01$ & $189 \pm 4$ \\
\hline $55 \%$ & $-2.41 \pm 0.03$ & $2.41 \pm 0.03$ (mass/pore) & $\mathrm{N} / \mathrm{A}^{b}$ & $\mathrm{~N} / \mathrm{A}^{b}$ \\
\hline \multicolumn{5}{|c|}{ Spruce, horizontal sector } \\
\hline $0 \%$ & $-3.89 \pm 0.01$ & $2.11 \pm 0.01$ (surface) & $2.74 \pm 0.02$ & $5300 \pm 90$ \\
\hline $10 \%$ & $-3.13 \pm 0.01$ & $2.87 \pm 0.01$ (surface) & $5.19 \pm 0.01$ & $1260 \pm 20$ \\
\hline $27 \%$ & $-2.80 \pm 0.01$ & $2.80 \pm 0.01$ (mass/pore) & $5.35 \pm 0.01$ & $393 \pm 6$ \\
\hline $49 \%$ & $-3.36 \pm 0.01$ & $2.64 \pm 0.01$ (surface) & $30.85 \pm 0.21^{a}$ & $30000 \pm 20000^{a}$ \\
\hline \multicolumn{5}{|c|}{ Beech, vertical sector } \\
\hline $0 \%$ & $-2.92 \pm 0.01$ & $2.92 \pm 0.01$ (mass/pore) & $4.60 \pm 0.02$ & $216 \pm 5$ \\
\hline $9 \%$ & $-2.41 \pm 0.01$ & $2.41 \pm 0.01$ (mass/pore) & $5.05 \pm 0.01$ & $127 \pm 3$ \\
\hline $26 \%$ & $-2.40 \pm 0.01$ & $2.40 \pm 0.01$ (mass $/$ pore) & $23.77 \pm 0.11^{a}$ & $1410 \pm 40^{a}$ \\
\hline $54 \%$ & $-2.28 \pm 0.02$ & $2.28 \pm 0.02($ mass $/$ pore $)$ & $27.12 \pm 0.25^{a}$ & $1400 \pm 100^{a}$ \\
\hline \multicolumn{5}{|c|}{ Oak, vertical sector } \\
\hline $0 \%$ & $-3.59 \pm 0.03$ & $2.41 \pm 0.03$ (surface) & $3.25 \pm 0.01$ & $29000 \pm 3000$ \\
\hline $10 \%$ & $-2.87 \pm 0.01$ & $2.87 \pm 0.01$ (mass/pore) & $5.33 \pm 0.01$ & $1630 \pm 50$ \\
\hline $26 \%$ & $-2.55 \pm 0.01$ & $2.55 \pm 0.01$ (mass/pore) & $5.72 \pm 0.01$ & $132 \pm 3$ \\
\hline $55 \%$ & $-2.23 \pm 0.01$ & $2.23 \pm 0.01$ (mass/pore) & $\mathrm{N} / \mathrm{A}^{b}$ & $\mathrm{~N} / \mathrm{A}^{b}$ \\
\hline \multicolumn{5}{|c|}{ Spruce, vertical sector } \\
\hline $0 \%$ & $-3.35 \pm 0.03$ & $2.65 \pm 0.03$ (surface) & $3.07 \pm 0.02$ & $43000 \pm 4000$ \\
\hline $10 \%$ & $-2.88 \pm 0.01$ & $2.88 \pm 0.01$ (mass/pore) & $5.49 \pm 0.01$ & $840 \pm 20$ \\
\hline $27 \%$ & $-2.75 \pm 0.01$ & $2.75 \pm 0.01$ (mass $/$ pore) & $5.57 \pm 0.01$ & $377 \pm 7$ \\
\hline $49 \%$ & $-3.08 \pm 0.01$ & $2.92 \pm 0.01$ (surface) & $31.85 \pm 0.21^{a}$ & $18000 \pm 4000^{a}$ \\
\hline
\end{tabular}

${ }^{a}$ The "reversed" Guinier-Porod model was fitted.

${ }^{b}$ Guinier-Porod model could not be fitted significantly.

Table 1: Radii of gyration and power-law exponents for carbonized wood samples (tangential cuts). Fitting of the power-law and a Guinier-Porod function was done simultaneously in the range $q \in[0.0086,0.576] \AA^{-1}$. The coefficient of determination $\left(R^{2}\right)$ was better than 0.995 for each fit.

a function of activation time. It is inferred therefore that the structural anisotropy of the studied activated carbons is rather complex, and has to be studied in at least two projections (in our case axial and tangential).

Although in our study loss of anisotropy was observed during activation, the extent of anisotropy may increase as well if the technological parameters are optimized. For example, the temperature and duration of carbonization and/or activation, or their consequent alternating application could result in improved anisotropy of the end-product. The existence of anisotropic features even after extensive burn-off might be possible in some cases.

Another means of optimization might be the choice of the precursors. Instead of phytogenic materials, other organic substances such as polymers can be used to achieve considerable anisotropy in the activated form by utilizing the original texture. Eventually, as the anisotropy of the carbon 
skeleton is coupled to that of the system of pores and voids, application of the nanometric anisotropy in the pore system in practice-e.g. in separation techniques - requires detailed study on the carbon skeleton, demonstrated in this study.

\section{Acknowledgements}

The underlying experimental measurements at Helmholtz-Zentrum Berlin, Electron storage ring BESSY were supported by the Seventh Framework Programme of the European Community (FP7/2007-2013) under grant agreement no. 226716. Measurements at HASYLAB/DESY were supported by grant agreement II-20080222 EC. We thank Prof. K. László for the possibility of adsorption measurements and Ms. E. Fülöp for the invaluable help in carrying them out. We would like to express our gratitude to Ms. K. Papp for the SEM images.

[1] Antal M, Grønli M. The art, science, and technology of charcoal production. Ind Eng Chem Res 2003;42(8):1619-40.

[2] Byrne C, Nagle D. Carbonization of wood for advanced materials applications. Carbon 1997;35(2):259-66.

[3] Byrne C, Nagle D. Carbonized wood monolithscharacterization. Carbon 1997;35(2):267-73.

[4] Kercher A, Nagle D. Microstructural evolution during charcoal carbonization by X-ray diffraction analysis. Carbon 2003;41:15-27.

[5] Paris O, Zollfrank C, Zickler G. Decomposition and carbonisation of wood biopolymers - a microstructural study of softwood pyrolisis. Carbon 2005;43:53-66.

[6] Fengel D, Wegener G. Wood-Chemistry, Ultrastructure, Reactions. Berlin, New York: De Gruyter; 1984.

[7] Fry S. The Growing Plant Cell Wall: Chemical and Metabolic Analysis. London: Longman; 1988.

[8] Kerr A, Goring D. Ultrastructural arrangement of the wood cell wall. Cellulose Chem Technol 1975;9:563-73.

[9] Sell J, Zimmermann T. Radial fibril agglomerations of the $\mathrm{S}_{2}$ on transverse-fracture surfaces of tracheids of tension-loaded spruce and white fir. Holz als Roh-und Werkstoff 1993;51(6):384-5.

[10] Booker R, Sell J. The nanostructure of the cell wall of softwoods and its functions in a living tree (Nanostruktur der Zellwand des Nadelholzes und ihre Funktion im lebenden Baum). Holz als Roh- und Werkstoff 1998;56(1):1-8.

[11] Harada H, Côté W. Structure of Wood. In: Biosynthesis and biodegradation of wood components (ed. T. Higuchi). Orlando: Acad. Press; 1985, p. 1-42.

[12] Lichtenegger H, Reiterer A, Stanzl-Tschegg S, Fratzl P. Variation of cellulose microfibril angles in softwoods and hardwoods - a possible strategy of mechanical optimization. Journal of Structural Biology 1999;128(3):257-69.

[13] Jakob H, Fratzl P, Tschegg S. Size and Arrangement of Elementary Cellulose Fibrils in Wood Cells: A SmallAngle X-Ray Scattering Study of Picea abies. J Struct Biol 1994;113:13-22.
[14] Jakob H, Fengel D, Tschegg S, Fratzl P. The Elementary Cellulose Fibril in Picea abies: Comparision of Transmission Electron Microscopy, Small-Angle X-ray Scattering, and Wide-Angle X-ray Scattering Results. Macromolecules 1995;28:8782-7.

[15] Sarén MP, Serimaa R, Andersson S, Saranpää P, Keckes J, Fratzl P. Effect of growth rate on mean microfibril angle and cross-sectional shape of tracheids in norway spruce. Trees 2004;18:354-62.

[16] Zimmermann $\mathrm{T}$, Thommen V, Reimann P, Hug H. Ultrastructural appearance of embedded and polished wood cell walls as revealed by Atomic Force Microscopy. Journal of Structural Biology 2006;156(2):363-9.

[17] Kataoka Y, Saiki H, Fujita M. Arrangement and superimposition of cellulose microfibrils in the secondary walls of coniferous tracheids. Mokuzai Gakkaishi 1992;38(4):327-35.

[18] Hult E, Larsson P, Iversen T. Cellulose fibril aggregationan inherent property of kraft pulps. Polymer 2001;42(8):3309-14.

[19] Fahlén J, Salmén L. On the lamellar structure of the tracheid cell wall. Plant Biology 2002;4(3):339-45.

[20] Salmén L. Micromechanical understanding of the cellwall structure. Comptes rendus-Biologies 2004;327(910):873-80.

[21] Rodríguez-Reinoso F, Molina-Sabio M, González MT. The use of steam and CO_2 as activating agents in the preparation of activated carbons. Carbon 1995;33(1):15-23.

[22] Wigmans T. Industrial aspects of production and use of activated carbons. Carbon 1989;27:13-22.

[23] Bóta A. Preparation and modification of activated carbon. Ph.D. thesis; Budapest University of Technology and Econommics; 1997.

[24] Bóta A, Szegedi K, Goerigk G, Haubold H. Fine structure of pyrolised and activated carbons prepared from different woods. In: HASYLAB Annual Report. 2002,.

[25] László K, Onyestyák G, Rochas C, Geissler E. Honeycomb carbon monoliths from Pinus sylvestris. Carbon $2005 ; 43: 2402-5$.

[26] Onyestyák G, Bóta A. Sorption dynamics of N_2 and O_2 in carbon monoliths from spruce, beech and oak affected by activation. Microporous and Mesoporous Materials 2009;120(1-2):84-90.

[27] Noszkó L, Bóta A, Simay Á, Nagy L. Preparation of activated carbon from the by-products of agricultural industry. Period Polytechn 1984;28(3-4):293-7.

[28] Bóta A. Modification of the structure of an active carbon by heat treatment. J Appl Cryst 1991;24(5):635-7.

[29] Paredes JI, Martínez-Alonso A, Tascón JMD. Application of scanning tunneling and atomic force microscopies to the characterization of microporous and mesoporous materials. Microporous and Mesoporous Materials 2003;65:93-126.

[30] Guinier A, Fournet G, Walker C, Yudowitch K. Small Angle X-ray Scattering. New York: John Wiley \& Sons, Inc.; 1955 , p. 30-2.

[31] Andersson S, Serimaa R, Torkkeli M, Paakkari T, Saranpää P, Pesonen E. Microfibril angle of Norway spruce [Picea abies (L.) Karst.] compression wood: comparison of measuring techniques. J Wood Sci 2000;46:343-9.

[32] Jakob HF, Tschegg SE, Fratzl P. Hydration Dependence of the Wood-Cell Wall Structure in Picea abies. A Small-Angle X-ray Scattering Study. Macromolecules 
1996;29:8435-40.

[33] Cave I. Theory of X-ray measurement of microfibril angle in wood. Wood Science and Technology 1997;31(4):225-34.

[34] Fratzl P, Fratzl-Zelman N, Klaushofer K, Vogl G, Koller $K$. Nucleation and growth of mineral crystals in bone studied by small-angle x-ray scattering. Calc Tissue Int 1991;48(6):407-13.

[35] Bale H, Schmidt P. Small-angle x-ray-scattering investigation of submicroscopic porosity with fractal properties. Phys Rev Lett 1984;53(6):596-9.

[36] Wong P, Bray A. Porod scattering from fractal surfaces. Phys Rev Lett 1988;60(13):1344-5.

[37] Schmidt PW. Small-angle scattering studies of disordered, porous and fractal systems. J Appl Cryst 1991;24:414-35.

[38] Hammouda B. A new Guinier-Porod model. J Appl Cryst 2010;43:716-9.

[39] Dubinin MM, Plavnik GM, Zaverina ED. Integrated study of the porous structure of active carbons from carbonized sucrose. Carbon 1964;2:261-8.

[40] Diduszko R, Swiatkowski A, Trznadel BJ. On surface of micropores and fractal dimension of activated carbon determined on the basis of adsorption and SAXS investigations. Carbon 2000;38(8):1153 -62.

[41] Pfeifer P, Rieker F, Ehrburger-Dolle TP, González MT, Hoffman WP, Molina-Sabio M, et al. Nearly spacefilling fractal networks of carbon nanopores. Phys Rev Lett 2002;88(11):115502-.

[42] Winter R, Gabke A, Czeslik C, Pfeifer P. Power-law fluctuations in phase-separated lipid membranes. Phys Rev E 1999;60:7354-9. 